\title{
Avaliação da Implantação do Checklist Perioperatório em um Hospital Público do
} Distrito Federal

\author{
Ana Cláudia Valentim Santana Pereira ${ }^{1}$ \\ Ruth Silva Matos ${ }^{2}$ \\ Marcelo Moreira Corgazinho ${ }^{3}$ \\ Gleyce Mikaelle Costa Quirino \\ Jacqueline Ramos de Andrade Antunes Gomes ${ }^{5}$ \\ Lauane Rocha Itacarambi ${ }^{6}$ \\ Cleber dos Santos Pinto ${ }^{7}$ \\ Erika Vanessa Silva Oliveira ${ }^{8}$ \\ Kerginaldo Severiano de Melo Junior ${ }^{9}$ \\ Osmar Pereira dos Santos ${ }^{10}$ \\ Fernanda Pereira de Oliveira ${ }^{11}$ \\ Jaqueline Dias Castelo Branco ${ }^{12}$
}

${ }_{\text {Enfermeira Residente }}^{1}$ do
ESCS/FEPECS/SES/DF

\section{RESUMO}

Introdução: A Organização Mundial de Saúde lançou em 2009 o Programa Cirurgias Seguras Salvam Vidas. Para alcançar os dez objetos essenciais propostos pela Organização Mundial de Saúde é necessária a utilização do Checklist de Cirurgia Segura. Objetivo: avaliar o grau da implantação do instrumento de checklist perioperatório de cirurgia segura em um Hospital Público do Distrito Federal. Método: trata-se de um estudo exploratório que avaliou a implantação de atividades relacionadas à aplicação do checklist no período Perioperatório, a 
partir da abordagem qualitativa. Resultados: participaram deste estudo 31 profissionais da Enfermagem que atuam no Centro Cirúrgico. A partir da matriz de avaliação foram pontuados os itens do checklist nos tempos Sing In, Time Out e Sing Out e para cada item da checagem foi estabelecida a pontuação de 10 pontos, totalizando 250 pontos (100\%). A pontuação obtida após análise dos questionários foi de 215 pontos (86\%). Conclusão: o checklist de segurança cirurgia segura realizado no Hospital Materno-Infantil de Brasília é considerado implantado. O preenchimento completo e correto do instrumento do checklist é o melhor meio existente para alcançar a real segurança do paciente cirúrgico.

Palavras chave:Centro Cirúrgico; Checklist Perioperatório; Segurança do Paciente.

\title{
Evaluation of the implementation of the perioperative checklist in a public hospital in the Federal District
}

\begin{abstract}
Introduction: The World Health Organization launched in 2009 the Safe Surgery Saves Lives Program. To achieve the ten essential objects proposed by the World Health Organization, it is necessary to use the Safe Surgery Checklist. Objective: to assess the degree of implementation of the perioperative checklist instrument for safe surgery in a Public Hospital in the Federal District. Method: this is an exploratory study that evaluated the implementation of activities related to the application of the checklist in the perioperative period, based on a qualitative approach. Results: 31 nursing professionals who work in the Surgical Center participated in this study. From the evaluation matrix, the checklist items were scored in the Sing In, Time Out and Sing Out times and for each check item a score of 10 points was established, totaling 250 points $(100 \%)$. The score obtained after analyzing the questionnaires were 215 points $(86 \%)$. Conclusion: the safe surgery safety checklist performed at the Hospital Materno-Infantil de Brasília is considered implemented. The complete and correct filling out of the checklist instrument is the best way to achieve real safety for the surgical patient.
\end{abstract}

Keywords: Surgical Center; Perioperative check-list; Patient safety.

\section{INTRODUÇÃO}

O cenário moderno e amplo dos sistemas de prestação de cuidados de saúde contrapõe-se as demandas ascendentes e aos recursos quase sempre insuficientes. A importante dimensão da qualidade do cuidado de saúde e a preocupação com a segurança do paciente constitui temática de relevância crescente entre os pesquisadores de todo o mundo ${ }^{1}$.

Desde a antiguidade, a responsável pelo ambiente seguro, limpo e confortável para a realização de cirurgias era a Enfermagem do Centro Cirúrgico (C.C). Até 1960 era voltada principalmente para a área instrumental onde as solicitações da equipe médica e as ações para o desenvolvimento do ato anestésico-cirúrgico eram atendidas, ou seja, assistência ao paciente 
cirúrgico ${ }^{2}$

Foi proposto em 1985 um modelo assistencial chamado de Sistema de Assistência de Enfermagem Perioperatória (SAEP) cuja finalidade era promover a assistência integral, continuada, participativa, individualizada, documentada e avaliada, onde o cliente é único e a assistência da enfermagem é uma ação integrada que envolve além da própria assistência a participação dos familiares na continuidade do cuidado ${ }^{2}$.

Para a Organização Mundial de Saúde (OMS) a Segurança do Paciente é alcançada através de três ações complementares: evitar a ocorrência dos eventos adversos, torna-los visíveis caso ocorram e minimizar suas consequências e efeitos com intervenções eficazes ${ }^{3}$.

Levando em consideração os avanços tecnológicos nas áreas clínicas das últimas décadas, a ampliação do acesso aos serviços de saúde ao longo dos anos e a difusão da informação a respeito da saúde, verifica-se que grandes transformações ocorreram no cenário dos sistemas de cuidados de saúde ${ }^{1}$.

Observa-se o aumento de iniciativas para a promoção da segurança e da qualidade na assistência à saúde em âmbito mundial, envolvendo desde a alta direção das instituições até seus colaboradores. Consequentemente, ocorre a otimização dos resultados com a meta de qualidade nos diversos serviços oferecidos a sociedade ${ }^{4}$.

Em 1991, os resultados de um Estudo da Prática Médica de Harvard, com foco na segurança do paciente, tornam evidente a preocupação com a magnitude da ocorrência de eventos adversos (EAs) ${ }^{1}$.

Define-se como Eventos Adversos (EA) os danos não intencionais que resultam na incapacidade temporária ou permanente e/ou prolongamento do tempo de internação na instituição de saúde ou até mesmo a morte como consequência de um cuidado de saúde prestado $^{4}$.

Esta mobilização é furto da constataçãode que a ocorrência de EA envolve 
consideráveis custos sociais e econômicos que pode implicar em danos irreversíveis aos pacientes e suas famílias. Assim, a partir dos anos 2000, a Segurança do Paciente passa a ser alvo de pesquisadores em todo o mundo como uma dimensão fundamental da qualidade em saúde ${ }^{1}$.

A Organização Mundial da Saúde (OMS) lançou em outubro de 2004 a Aliança Mundial para a Segurança do Paciente, com o intuito de facilitar o desenvolvimento de uma política para aprimorar a Segurança do Paciente e a qualidade dos serviços de saúde nos estados membros. Essas ações foram organizadas através de campanhas de segurança denominadas desafios globais para a segurança do paciente, onde inicialmente apresentavam três desafios: uma assistência limpa é uma assistência mais segura, cirurgias seguras salvam vidas e prevenção da resistência aos antimicrobianos ${ }^{5}$.

Em 2008, a OMS instituiu a campanha "Cirurgias Seguras Salvam Vidas", que pretendia diminuir a ocorrência de danos aos pacientes cirúrgicos e definir padrões de segurança aplicados em qualquer país membro. Este documento foi adaptado pela Agência Nacional de Vigilância Sanitária (ANVISA) e amplamente divulgado no Brasil ${ }^{5}$.

Sendo assim, foi proposta uma lista de Verificação de Segurança Cirúrgica (checklist) para ser empregada em qualquer hospital independente do seu grau de complexidade de atuação, com o objetivo de ajudar as equipes cirúrgicas a seguirem os passos críticos de segurança de forma embasada e sistemática ${ }^{5}$.

O checklist Perioperatório consiste em 19 itens divididos em três momentos: a) Identificação ou SignIn (antes da indução anestésica): verifica-se identidade do paciente, o procedimento e o local da cirurgia e se o consentimento para o procedimento foi assinado. Verifica-se lateralidade, funcionamento de equipamentos e se existem etapas críticas na anestesia. b) Confirmação ou Time Out (antes da incisão na pele-pausa cirúrgica): Faz-se a apresentação da equipe, a conferência da identidade do paciente, do procedimento e da 
lateralidade. A equipe revisa pontos críticos da cirurgia, confirma o antibiótico profilático, além da certificação da disponibilidade de exames de imagem e laboratoriais. c) Registro ou Sign out (antes do paciente sair da sala cirúrgica): Analisa-se o procedimento, é realizada a contagem de compressas e instrumentais, identificação de peças anatômicas, conferência dos equipamentos utilizados, elabora-se o plano de cuidados em relação ao pós-operatório, antes de encaminhar o paciente para Sala de Recuperação Pós Anestésica ${ }^{6}$.

Um dos principais objetivos da Organização Mundial de Saúde (OMS) através da campanha Cirurgia Segura inclui reduzir a mortalidade dos pacientes cirúrgicos, proporcionando as equipes e aos administradores hospitalares, informações e orientações sobre a função de cada profissional e qual o padrão de um procedimento cirúrgico seguro, além de disponibilizar um instrumento de avaliação único do serviço para Vigilância Nacional e Internacional ${ }^{6}$

Após essa década ocorreu um grande avanço das técnicas cirúrgicas e instrumentais tornando assim, as cirurgias mais complexas, o que provocou na enfermagem a necessidade da formação de um conhecimento e de uma fundamentação científica que lhe desse base e identidade ${ }^{2}$.

Inserido nesta temática a estimativa mundial mostrou que metade das complicações com pacientes pós operados eram evitáveis, destacando o potencial previsível de dano. Óbitos e sequelas decorrentes de erros da assistência contribuíram para uma maior conscientização mundial, afim de promover a segurança do paciente, visando uma redução do risco de dano desnecessário ao mínimo aceitáve ${ }^{7}$.

Foi feito um estudo multicêntrico internacional que evidenciou uma redução de $36 \%$ das complicações e $47 \%$ de mortes em pacientes cirúrgicos com a implementação e adesão do checklist cirúrgico ${ }^{3}$.

Levando em conta as informações acima citadas e as constantes atualizações acerca do 
tema, o presente trabalho objetiva avaliar a implantação do checklist perioperatório em um centro cirúrgico de um hospital público do Distrito Federal, e identificar as possíveis barreiras para sua utilização efetiva e ajustar o seu uso para garantir a segurança do paciente.

\section{MATERIAL E MÉTODO}

Trata-se de um estudo exploratório que avaliou a implantação de atividades relacionadas à aplicação do checklist no período Perioperatório, a partir da abordagem qualitativa. Com esse estudo a avaliação formativa buscou fornecer um feedback afim de ajustar o processo da implantação do checklist para a melhoria do serviço. A avaliação do processo de uma intervenção busca a adequação dos meios e recursos utilizados perante os resultados parciais ou finais, referenciados pelas metas e indicadores da intervenção ${ }^{8}$.

A população foi constituída pela equipe de enfermagem - enfermeiros e técnicos de enfermagem - que atuam no centro cirúrgico do Hospital Materno Infantil de Brasília (HMIB). A coleta dos dados foi realizada através de uma matriz de julgamento (quantitativo). A pesquisa quantitativa significa quantificar opiniões e dados na forma de coleta de informações, assim como o emprego de recursos e técnicas estatísticas desde as mais simples (Ex: porcentagens, coeficientes de correlação, análise de regressão etc), geralmente utilizados em defesas de teses 9 .

Atuam na unidade do centro cirúrgico uma equipe multiprofissional que inclui equipe médica, enfermeiros gerentes, enfermeiros assistenciais, técnicos de enfermagem, residentes da residência em medicina e da residência multiprofissional, estudantes de graduação em medicina e enfermagem, além de funcionários administrativos e equipe de serviços gerais. No entanto, apesar da equipe cirúrgica ser responsável pela segurança do paciente, a responsabilidade pela execução das principais etapas da aplicação do preenchimento do checklist é da equipe da enfermagem.

A pesquisa foi realizada no Centro Cirúrgico do Hospital Materno Infantil de Brasília 
(HMIB), considerado um hospital de referência de cirurgia pediátrica no Distrito Federal. Foi inaugurado no dia 22 de novembro de 1966, porém seu funcionamento se deu em 02 de janeiro de 1967, com 5.325m2 e capacidade inicial de 150 leitos. Este hospital dedica 100\% dos seus leitos ao Sistema Único de Saúde (SUS). Posteriormente, foi ampliado para 270 leitos com uma área física de 19.299,20m2. Com essa nova extensão, o Hospital direcionou o enfoque clinico para o atendimento à saúde da mulher e da criança, buscando investir no parto sem intervenção cirúrgica. O Centro Cirúrgico do hospital dispõe de 05 salas cirúrgicas, sendo 04 salas destinadas a cirurgias eletivas e 01 para as cirurgias de emergência. A Sala de Recuperação Pós Anestésica dispõe de 06 leitos. São 38 funcionários da equipe de enfermagem.

Participaram deste estudo 31 profissionais da Enfermagem que atuem no Centro Cirúrgico. A amostra corresponde em $88 \%$ do total de profissionais. Foram incluídos os profissionais que atuam nas unidades de Centro Cirúrgico descritas como cenários investigativos do estudo, que estivessem no Centro Cirúrgico no momento do preenchimento do checklist e que participassem de todo o processo Perioperatório. Para exclusão o critério foi estar afastado do cenário durante o período de coleta de dados por período de férias, licenças médicas, ou simplesmente por questões de escala diária de trabalho.

A pesquisa quantitativa foi realizada por meio de uma Matriz de Avaliação adaptada do "Checklist da Campanha de Cirurgia Segura OMS" e terá perguntas objetivas. Esse instrumento de pesquisa contará com uma Matriz de Relevância e Julgamento (Apêndice I) e haverá pontuação para os itens mais relevantes (de 1 a 3), onde 1 representa baixa relevância, 2 média relevância e 3 alta relevância.

Este instrumento é primordial para o processo de julgamento fidedigno e transparente. Outro ponto essencial é a descrição dos indicadores para avaliar o seu grau de implantação alcançado pela intervenção, qualificados estes, através da pontuação de cada atividade 
segundo a sua relevância. Assim, durante a aplicação do instrumento, os pesquisadores poderão tomar nota sobre as possíveis variáveis contextuais que influenciam na implantação da atividade, como os recursos humanos, recursos materiais, apoio institucional, dentre outros.

Os parâmetros de julgamento da implantação da atividade serão definidos da seguinte forma ${ }^{8}$ :

- $\quad$ Acima de $75 \%$ considera-se na ação implantada;

- $\quad$ Entre 50 e $75 \%$ parcialmente implantada;

- $\quad$ Entre 25 e $49 \%$ implementação incipiente; e

- $\quad$ Abaixo de $25 \%$ atividade não implantada.

Para melhor visualização e compreensão dos valores, os dados foram apresentados em Tabelas que seguiram o modelo do instrumento de avaliação, para melhor visualização e compreensão dos valores.

O projeto foi submetido a análise do Comitê de Ética da Fundação de Ensino e Pesquisa em Ciências da Saúde (FEPECS), conforme preconizado pela Resolução 466/2012 CONEP/CNS/MS, e foi aprovado com as seguintes especificações: CAAE número 01535118.2.0000.5553 e Parecer número 2.995.131. Os participantes desta pesquisa assinaram o Termo de Consentimento Livre e Esclarecido (TCLE) e os dados relacionados aos pacientes e equipe multidisciplinar terão seu anonimato assegurado através da codificação no referido instrumento.

\section{RESULTADOS}

Os dados coletados do questionário utilizado na pesquisa foram consolidados e organizados de acordo com os tempos cirúrgicos: a) Antes da indução anestésica (Sing In); b) Antes de iniciar a cirurgia (Time Out); c) Antes do paciente sair da sala cirúrgica (Sing Out).

Tabela 1- Perfil dos participantes da pesquisa 


\begin{tabular}{|c|c|c|}
\hline & (n. 31) & Porcentagem \\
\hline Total- número & 31 & $100 \%$ \\
\hline \multicolumn{3}{|l|}{$\underline{\text { Sexo }}$} \\
\hline Masculino & 07 & $23 \%$ \\
\hline Feminino & 24 & $77 \%$ \\
\hline \multicolumn{3}{|l|}{ Faixa Etária } \\
\hline 20-40 anos & 16 & $52 \%$ \\
\hline 40-60 anos & 15 & $48 \%$ \\
\hline \multicolumn{3}{|l|}{ Escolaridade } \\
\hline Ensino Médio & 12 & $39 \%$ \\
\hline Ensino Superior & 13 & $42 \%$ \\
\hline Pós- Graduação & 06 & $19 \%$ \\
\hline \multicolumn{3}{|l|}{ Cargo } \\
\hline Técnico de Enfermagem & 05 & $16 \%$ \\
\hline Enfermeiro & 26 & $84 \%$ \\
\hline \multicolumn{3}{|l|}{ Tempo de Atuacão no C.C } \\
\hline 0-10 anos & 18 & $58 \%$ \\
\hline 10-20 anos & 09 & $29 \%$ \\
\hline Mais de 20 anos & 04 & $13 \%$ \\
\hline
\end{tabular}

Fonte: Elaborada pelo autor.

Observa-se a partir dos resultados expressos na Tabela 1, que a maioria dos participantes era do sexo feminino, com $77 \%$ dos profissionais, enquanto de $23 \%$ eram do sexo masculino. Pode-se observar que 52\% encontravam-se entre a faixa etária de 20 a 40 anos e $48 \%$ entre 40 a 60 anos.

Em relação à escolaridade, 39\% dos profissionais possuem ensino médio completo, $42 \%$ ensino superior completo e $19 \%$ nível superior completo. Em relação à categoria profissional, pode-se observar que $84 \%$ eram técnicos de enfermagem enquanto que $16 \%$ enfermeiros. Quanto ao tempo de atuação em centro cirúrgico, 58\% dos profissionais atuavam entre 0 a 10 anos, $29 \%$ entre 10 a 20 anos, e $4 \%$ acima de 20 anos.

Para análise dos dados referente à implantação do checklist de cirurgia segura, foi atribuído um valor e 10 pontos para cada item a ser analisado, onde se espera que essa pontuação seja alcançada, para assim constatar a implementação ou não do checklist de cirurgia segura nos seus três tempos.

Tabela 2- Checklist de cirurgia segura considerando a etapa Antes da Indução Anestésica (Check in) 


\begin{tabular}{|c|c|c|c|c|}
\hline \multicolumn{5}{|c|}{$\begin{array}{l}\text { Checklist de Cirurgia Segura } \\
\text { ** Antes da Indução Anestésica (Sing-In) }\end{array}$} \\
\hline & $\begin{array}{l}\text { Pontuação } \\
\text { Atribuída }\end{array}$ & $\begin{array}{l}\text { Pontuação } \\
\text { Esperada }\end{array}$ & $\begin{array}{l}\text { Pontuação } \\
\text { Obtida }\end{array}$ & $\begin{array}{l}\text { Grau de } \\
\text { Implantação } \\
(\%)\end{array}$ \\
\hline Identificação do paciente & 10 & 10 & 8,4 & $*$ Acima de \\
\hline Local da cirurgia a ser feita & 10 & 10 & 8,4 & $\begin{array}{l}75 \% \\
\text { considera-se }\end{array}$ \\
\hline Procedimento a ser realizado & 10 & 10 & 9,0 & $\begin{array}{l}\text { na ação } \\
\text { implantada; }\end{array}$ \\
\hline $\begin{array}{l}\text { Consentimento } \\
\text { realizado }\end{array}$ & 10 & 10 & 8,6 & $\begin{array}{l}* \text { Entre } 50 \text { e } \\
75 \% \\
\text { parcialmente }\end{array}$ \\
\hline Sítio cirúrgico do lado correto & 10 & 10 & 8,4 & $\begin{array}{l}\text { implantada; } \\
\text { *Entre } 25 \text { e }\end{array}$ \\
\hline $\begin{array}{l}\text { Checagem do equipamento } \\
\text { anestésico }\end{array}$ & 10 & 10 & 9,2 & $\begin{array}{l}49 \% \\
\text { implementação } \\
\text { incipiente; e }\end{array}$ \\
\hline Oxímetro de pulso instalado & 10 & 10 & 10 & $\begin{array}{l}\text { *Abaixo de } \\
25 \% \text { atividade }\end{array}$ \\
\hline Paciente tem alguma alergia? & 10 & 10 & 8,5 & $\begin{array}{l}\text { não } \\
\text { implantada }\end{array}$ \\
\hline $\begin{array}{l}\text { Há risco de via aérea } \\
\text { difícil/broncoaspiração? }\end{array}$ & 10 & 10 & 8,2 & \\
\hline $\begin{array}{l}\text { Há risco de perda sanguínea>de } \\
500 \mathrm{ml} ?\end{array}$ & 10 & 10 & 8,0 & \\
\hline Total & 100 & 100 & 86,7 & $86,7 \%$ \\
\hline
\end{tabular}

Fonte: Elaborada pela autora

Pode-se observar pela Tabela 2, que durante o período que antecede a indução anestésica (Sing In), no tocante à confirmação da identificação do paciente, foi obtida nota de 8,4 dos 10 pontos esperados, seguidos pela confirmação do local da cirurgia a ser feita, com nota de 8,4; confirmação do procedimento a ser realizado, nota de 9,0; confirmação da presença do termo de consentimento sobre o procedimento a ser realizado, nota de 8,6; confirmação do sítio cirúrgico e lateralidade do procedimento a ser realizado, nota de 8,4; confirmação da checagem do equipamento anestésico, nota de 9,2; confirmação da instalação e funcionamento do oxímetro de pulso, nota de 10; confirmação da existência de alergias, 
nota de 8,5; confirmação da existência de via aérea difícil e broncoaspiração, nota de 8,2; e confirmação do risco de perda sanguínea maior de $500 \mathrm{ml}$, nota de 8,0 dos 10 pontos esperados.

Tabela 3- Checklist de cirurgia segura considerando a etapa Antes de Iniciar a Cirurgia (Time Out)

\begin{tabular}{|c|c|c|c|c|}
\hline \multicolumn{5}{|c|}{$\begin{array}{l}\text { Checklist de Cirurgia Segura } \\
* * \text { Antes de Iniciar a Cirurgia (Time Out) }\end{array}$} \\
\hline & $\begin{array}{l}\text { Pontuação } \\
\text { Atribuída }\end{array}$ & $\begin{array}{l}\text { Pontuação } \\
\text { Esperada }\end{array}$ & $\begin{array}{l}\text { Pontuação } \\
\text { Obtida }\end{array}$ & $\begin{array}{l}\text { Grau de } \\
\text { Implantação } \\
(\%)\end{array}$ \\
\hline $\begin{array}{l}\text { Todos os profissionais da equipe } \\
\text { confirmam seus nomes e } \\
\text { profissões }\end{array}$ & 10 & 10 & 7,2 & $\begin{array}{l}\text { *Acima de } \\
75 \% \\
\text { considera-se }\end{array}$ \\
\hline $\begin{array}{l}\text { O cirurgião, o anestesista e a } \\
\text { enfermagem } \\
\text { confirmam: } * \text { verbalmente } \\
\text { paciente }\end{array}$ & 10 & 10 & 8,3 & $\begin{array}{l}\text { na ação } \\
\text { implantada; } \\
\text { *Entre } 50 \text { e } \\
75 \%\end{array}$ \\
\hline *Local da cirurgia a ser feita & 10 & 10 & 8,0 & parcialmente \\
\hline *Procedimento a ser realizado & 10 & 10 & 8,0 & implantada; \\
\hline $\begin{array}{l}\text { Antecipação de eventos críticos: } \\
\text { *Revisão do cirurgião: há passos } \\
\text { críticos na cirurgia? Qual a sua } \\
\text { duração estimada? Há possíveis } \\
\text { perdas sanguíneas? }\end{array}$ & 10 & 10 & 8,9 & $\begin{array}{l}\text { *Entre } 25 \text { e } \\
49 \% \\
\text { implementação } \\
\text { incipiente; e } \\
\text { *Abaixo de }\end{array}$ \\
\hline $\begin{array}{l}\text { *Revisão do anestesista: há } \\
\text { alguma preocupação em relação } \\
\text { ao paciente? }\end{array}$ & 10 & 10 & 10 & $\begin{array}{l}25 \% \text { atividade } \\
\text { não } \\
\text { implantada }\end{array}$ \\
\hline $\begin{array}{l}\text { * Revisão da enfermagem: } \\
\text { Houve correta esterilização do } \\
\text { instrumental cirúrgico? }\end{array}$ & 10 & 10 & 10 & \\
\hline $\begin{array}{l}\text { Há alguma preocupação em } \\
\text { relação aos equipamentos? }\end{array}$ & 10 & 10 & 8,3 & \\
\hline $\begin{array}{l}\text { O antibiótico profilático foi dado } \\
\text { nos últimos } 60 \text { minutos? }\end{array}$ & 10 & 10 & 7,6 & \\
\hline $\begin{array}{l}\text { Exames de imagens estão } \\
\text { disponíveis? }\end{array}$ & 10 & 10 & 9,0 & \\
\hline Total & 100 & 100 & 85,3 & $85,3 \%$ \\
\hline
\end{tabular}

Fonte: Elaborada pela autora

Pode-se observar a partir da Tabela 3, que durante o período que antecede o início do procedimento cirúrgico (Time Out), no que se refere a confirmação verbal da identificação da equipe e suas respectivas profissões, foi obtida uma nota de 7,2 dos 10 pontos esperados; 
seguido por confirmação verbal pela enfermagem, cirurgião e anestesista a respeito da identificação do paciente, nota de 8,3; confirmação verbal do local da cirurgia a ser realizada, nota de 8,0; confirmação verbal do procedimento a ser realizado, nota de 8,0 ; confirmação verbal da antecipação dos eventos críticos, nota de 8,9; confirmação verbal da revisão do anestesista, nota de 10; confirmação verbal da revisão da enfermagem, nota de 10; preocupação em relação aos equipamentos, nota de 8,3; confirmação da administração do antibiótico profilático, nota de 7,6; e existência de exames de imagem disponíveis, nota de 9,0 dos 10 pontos esperados.

Tabela 4- Checklist de cirurgia segura considerando a etapa antes do paciente sair da sala cirúrgica (CheckOut)

\begin{tabular}{|c|c|c|c|c|}
\hline $\begin{array}{l}\text { Checklist de Cirurgia Segura } \\
* * \text { Antes do paciente sair da sala }\end{array}$ & irúrgica ( & g Out) & & \\
\hline & $\begin{array}{l}\text { Pontuação } \\
\text { Atribuída }\end{array}$ & $\begin{array}{l}\text { Pontuação } \\
\text { Esperada }\end{array}$ & $\begin{array}{l}\text { Pontuação } \\
\text { Obtida }\end{array}$ & $\begin{array}{l}\text { Grau de } \\
\text { Implantação (\%) }\end{array}$ \\
\hline $\begin{array}{l}\text { A enfermeira } r \text { confirma } \\
\text { verbalmente com a equipe: } \\
\text { * Nome do procedimento } \\
\text { realizado }\end{array}$ & 10 & 10 & 7,6 & $\begin{array}{l}\text { *Acima de } 75 \% \\
\text { considera-se na } \\
\text { ação implantada; } \\
\text { *Entre } 50 \text { e } 75 \%\end{array}$ \\
\hline $\begin{array}{l}\text { *A contagem de compressas, } \\
\text { instrumentos e agulhas está } \\
\text { correta }\end{array}$ & 10 & 10 & 9,8 & $\begin{array}{l}\text { parcialmente } \\
\text { implantada; } \\
\text { *Entre } 25 \text { e } 49 \% \\
\text { implementação }\end{array}$ \\
\hline $\begin{array}{l}\text { *Biópsias estão identificadas } \\
\text { com o nome do paciente }\end{array}$ & 10 & 10 & 10 & $\begin{array}{lr}\text { incipiente; e } \\
\text { *Abaixo de } & 25 \% \\
\text { atividade } & \text { não }\end{array}$ \\
\hline $\begin{array}{l}\text { *Houve algum problema com } \\
\text { os equipamentos que deve ser } \\
\text { resolvido }\end{array}$ & 10 & 10 & 8,4 & implantada. \\
\hline $\begin{array}{l}\text { *O cirurgião, o anestesista e a } \\
\text { enfermagem analisam os pontos } \\
\text { mais importantes na } \\
\text { recuperação pós-anestésica e } \\
\text { pós-operatória desse paciente }\end{array}$ & 10 & 10 & 7,2 & \\
\hline TOTAL & 50 & $\begin{array}{l}50 \\
(100 \%)\end{array}$ & 43 & $86 \%$ \\
\hline
\end{tabular}


Diante da Tabela 4, observa-se que durante o período que antecede a saída do paciente da sala operatória (Sing Out), no que se refere a confirmação verbal do nome do procedimento realizado, obteve-se nota de 7,6 dos 10 pontos esperados; seguidos pela contagem de compressas, instrumentos e agulhas, nota de 9,8; identificação das biópsias, nota de 10; resolução de algum problema com equipamentos, nota de 8,4 ; e análise feita pela equipe dos pontos mais importantes na recuperação pós anestésica e pós operatória, nota de 7,2 dos 10 pontos esperados.

Foi contatado que os profissionais que mais se mostram resistentes em participar do preenchimento correto do checklist foram os cirurgiões e os anestesistas.

Neste instrumento de avaliação acima descrito, foram pontuados os itens do checklist nos tempos Sing In, Time Out e Sing Out e para cada item da checagem foi estabelecida a pontuação de 10 pontos, totalizando assim, 250 pontos (100\%). A pontuação obtida após análise dos questionários foi de 215 pontos (86\%). Nesse sentido, os parâmetros de julgamento da implantação da atividade foram definidos com referência ao percentual acima de $75 \%$, que considera a atividade como implantada no Centro Cirúrgico analisado.

\section{DISCUSSÃO}

No que diz respeito à segurança do paciente, depara-se com a questão da cultura de segurança, que é conhecida como "resultado das atitudes, percepções e competências, e dos padrões de comportamento e valores de um grupo, que irá determinar o compromisso, o estilo e a competência de uma organização de saúde, e de uma gestão de segurança"10.

Neste contexto, a mudança cultural acerca da segurança do paciente é fundamental para que sejam implementadas medidas eficientes de prevenção e redução de riscos e EA. Torna-se necessária então, a construção de uma nova cultura que entenda os valores, normas e crenças daquilo que é importante em uma instituição, além de quais atitudes e 
comportamentos relacionados a segurança do paciente são recompensados, suportados e $\operatorname{esperados}^{10}$.

Busca-se instituir a segurança nas organizações de saúde enquanto processo cultural, ao estimular a maior consciência dos profissionais quanto a cultura de segurança, compromisso ético no gerenciamento de risco, adquirindo assim, maior segurança para si e para a clientela atendida no que desrespeito a qualidade da assistência prestada ${ }^{4}$.

Tornou-se urgente a redução de eventos adversos em todo o mundo, que são normalmente associados a um erro individual humano, porém deve-se considerar também como desencadeadores as próprias condições de trabalho, os aspectos estruturais e a complexidade das atividades desenvolvidas ${ }^{4}$. No que tange à assistência em saúde nos hospitais brasileiros, acredita-se que esses erros e suas consequências ao paciente são numericamente maiores, por causa da precariedade dos serviços prestados, carga horário excessiva, má remuneração dos profissionais e a falta de dimensionamento adequado de recursos e de pessoal ${ }^{4}$.

No entanto, estratégias simples e eficazes podem prevenir e reduzir os riscos e danos nos serviços de saúde, por meio da implementação e segmento de protocolos específicos, associados as barreiras de segurança nos sistemas e a educação permanente. A aplicação deste conhecimento é importante para estabelecer articulações entre os serviços hospitalares, promover ações de educação em saúde, auxiliando na redução da mortalidade associada a eventos adversos graves e melhorar a qualidade de vida dos profissionais e clientes ${ }^{4}$.

O sistema de assistência à saúde não pode combater o fator humano com todas as suas possibilidades de variação e falibilidade. Essas condições inerentes a prestação do serviço não podem ser totalmente alteradas e controladas, porém é possível definir estratégias preventivas por meio de adequações no processo de trabalho afim de evitar eventos adversos, garantir a melhoria da qualidade e aumentar a segurança do paciente ${ }^{11}$. 
A clara necessidade de se criar controles e padrões de segurança para a assistência em saúde embasou a importação do comprovado método sistematizado de verificação de segurança da interação homem e equipamentos, ou seja, a utilização de um checklist prévio a cada procedimento realizado. A maior relevância deste processo se baseia não só na interação homem e tecnologia, mas também e de maneira significante, na interação e comunicação entre os profissionais da equipe com os pacientes ${ }^{11}$.

No entanto, no que diz respeito à assistência à saúde, o primeiro princípio a ser considerado é a variabilidade, ou seja, não há um único padrão de paciente ou de recursos estruturais, nem normas institucionais e equipes que atendam de forma igualitária. Cada instituição de saúde tem a sua própria realidade e contexto, sendo necessário assim, que cada "time" entenda a variabilidade do seu ambiente e evolua no sentido de padronizar suas ações ao máximo possível em um cenário onde cada procedimento detém suas particulariedades ${ }^{11}$.

Os recursos materiais e o aparato tecnológico necessários à execução de um procedimento cirúrgico estão associados a interação e comunicação dos indivíduos, serviços e equipamentos. Somente a habilidade técnica dos profissionais que atuam em centro cirúrgico não é suficiente, mas também, senão mais importante, a capacidade de se comunicar de forma eficaz, reconhecendo suas limitações, aprendendo com os seus erros e principalmente de trabalhar em equipe, é o que garante a melhoria contínua da qualidade e segurança da assistência ao paciente ${ }^{11}$.

O checklist de cirurgia segura nasce do contexto com a preocupação com a segurança do paciente em todas as organizações de saúde, e aparece como uma ferramenta com grande potencial para orientar e coordenar a assistência cirúrgica, promover a comunicação e união da equipe, incentivar o desenvolvimento da cultura e segurança e contribuir para a redução do número de complicações evitáveis durante e/ou após os procedimentos ${ }^{12}$. É baseado em três princípios: simplicidade, ampla aplicabilidade e possibilidade de mensuração do impacto, 
norteando as equipes para o cumprimento eficiente das etapas críticas de segurança, minimizando os riscos evitáveis que possam colocar em risco o bem-estar e a vida dos clientes cirúrgicos ${ }^{3}$

O objetivo principal estabelecido pela Organização Mundial de Saúde (OMS) é diminuir a morbimortalidade em paciente cirúrgicos, oferecendo as equipes cirúrgicas e administradores hospitalares diretrizes sobre a função de cada indivíduo e do que se entende como padrão de uma cirurgia segura. Tem como objetivo também, disponibilizar um instrumento de avaliação uniforme do serviço para a vigilância nacional e internacional ${ }^{13}$.

O emprego desta ferramenta tem sido muito recomendado como intervenção efetiva, de aplicação relativamente fácil e de baixo custo. Estudos realizados em países desenvolvidos comprovam que a atualização do checklist diminui as taxas de mortalidade e de complicações entre pacientes cirúrgicos, como também, diminui o número de erros ocasionados por falhas de comunicação entre os membros da equipe ${ }^{12}$.

A utilização do checklist está atrelado a sistematização de dados para identificação de pontos que possam ser reforçados ou alterados visando a melhoria nos padrões de cuidado, para redução das taxas de morbimortalidade, de complicações cirúrgicas, prevenção de infecções e a redução do número de erros ocasionados pela simples falha de comunicação da equipe $^{11}$.

Estima-se que são necessários por volta de 3 minutos para a aplicação das 3 fases do processo de verificação do checklist e o ideal é que tenha apenas um responsável para esta verificação, sendo o enfermeiro o profissional normalmente indicado para a checagem, porém qualquer membro da equipe multiprofissional que participa do procedimento cirúrgico pode ser o coordenador desta verificação. O importante é que este profissional tenha plena autoridade sobre o processo, podendo interromper o procedimento cirúrgico se julgar insatisfatório alguns dos itens do instrumento, mesmo que esta interrupção cause algum 
desgaste perante a equipe. Pois ao ocorrer erro na checagem todo o processo terá sido em vão colocando o paciente em risco ${ }^{13}$.

A existência de incoerência na checagem de segurança ou falta de preenchimento correto sugere orientação inadequada sobre o checklist, sua importância e objetivo, além de comprovar a existência de fragilidade na comunicação entre os profissionais envolvidos e pouca valorização da ferramenta. Estas incoerências devem ser avaliadas cuidadosamente afim de se tornar objetivo de intervenções futuras ${ }^{12}$.

A importância da adesão ao checklist se justifica pela complexidade do ambiente do centro cirúrgico, ambiente este onde se encontram muitos profissionais de diversas especialidades e esta equipe está sujeita a déficit de memória e de atenção, ainda que em questões rotineiras, onde facilmente podem ser negligenciadas ${ }^{12}$.

Experiências comprovam que o sucesso da implantação do checklist e de bons resultados estão relacionados a participação, envolvimento e engajamento das equipes. Considerando, ainda, que o relacionamento interpessoal é o segundo item apontado como gatilho do estresse em profissionais do centro cirúrgico, atrás somente da sobrecarga de trabalho, o checklist é visto como um facilitador na assistência ao paciente e visa diminuir o atrito provocado por situações não planejadas, a apresentação dos membros da equipe e a melhoria da segurança para o paciente ${ }^{13}$.

Cabe destacar que a utilização do checklist por si só não configura uma única solução capaz de promover uma assistência cirúrgica segura ao paciente. Para que essa ferramenta seja considerada um instrumento real de transformação na assistência cirúrgica, é necessário investir no desenvolvimento de estratégias que promovam uma cultura de segurança do paciente, envolvendo gestores e profissionais de saúde, bem como pacientes, e não apenas os cirurgiões responsáveis pelos procedimentos. Também, é de extrema importância realizar avaliações periódicas da adesão ao checklist e oferecer feedbacks para as equipes cirúrgicas 
sobre os indicadores da efetividade da aplicação do instrumento na redução de complicações $^{12}$.

Em se tratando de Centro Cirúrgico (CC) a equipe multidisciplinar precisa trabalhar em conjunto e de forma eficiente, além de utilizar todo o conhecimento disponível em prol do paciente a fim de evitar danos ao mesmo. O cotidiano deste tipo de ambiente requer profissionais capacitados tecnicamente, atentos aos procedimentos padrão e em constante comunicação, com foco na redução do número de erros, do estresse da atividade no setor que possam resultar na redução da eficácia da equipe ${ }^{10}$.

Contudo, a implementação do checklist é um passo novo em direção a construção de uma nova cultura de segurança na sala cirúrgica, tanto para o paciente, equipe cirúrgica e para própria instituição de saúde ${ }^{6}$.

Sendo assim, não basta que as instituições proponham protocolos, é necessário que os profissionais façam uso desta ferramenta apresentada, que as equipes compreendam a importância e a necessidade, aceitem o processo e incorporem as práticas diárias. O essencial para o sucesso do procedimento é realizar a checagem por meio de um coordenador com a participação do paciente e, principalmente de toda equipe multidisciplinar ${ }^{13}$.

\section{CONSIDERAÇÕES FINAIS}

A análise dos dados levantados demonstra que a equipe do centro cirúrgico tem tido o cuidado de não deixar de preencher a folha do checklist de cirurgia segura de maneira adequada e, de acordo com a pontuação estabelecida de 250 pontos esperados; foram alcançados 215 pontos, o que corresponde a $86 \%$ de implantação do instrumento. Portanto, o checklist de segurança cirurgia segura realizado no Hospital Materno-Infantil de Brasília é considerado implantado.

No momento do preenchimento é necessária uma comunicação eficiente e completa 
entre os profissionais envolvidos para que todos os itens do checklist de cirurgia segura sejam conferidos e preenchidos. Somente assim, seus efeitos poderão ser efetivos e contínuos no que diz respeito a segurança do paciente frente ao procedimento realizado.

O preenchimento completo e correto do instrumento do checklist é o melhor meio existente para alcançar a real segurança do paciente, além de ser a única maneira de se monitorar a qualidade e a eficácia e a qualidade do tratamento cirúrgico e a responsabilidade dos profissionais envolvidos.

Embora se perceba que um número considerável de integrantes da equipe multidisciplinar do centro cirúrgico zele por um preenchimento correto, claro e livre de erros do instrumento, encontramos ainda aspectos que precisam e podem ser melhorados no que diz respeito ao cumprimento integral de todos os itens englobados no checklist.

No cenário deste estudo, a principal barreira descrita pela equipe à utilização do checklist foi a resistência por parte dos cirurgiões e anestesistas, para proceder a confirmação verbal dos itens de segurança de sua responsabilidade, mostrando assim, a necessidade de investimentos no conhecimento em relação ao instrumento utilizado.

Depreende-se dos dados levantados que existe também uma falta de compromisso profissional e até uma certa dose de desinteresse com relação a utilização do checklist, uma vez que o seu preenchimento acaba sendo feito de forma automática e descompromissada apenas "para se cumprir uma rotina", uma vez que certos itens do instrumento podem parecer óbvios e desnecessários diante de toda complexidade do procedimento a ser realizado.

Sendo necessário então, cada vez mais, a implementação de ações para potencializar e desenvolver uma cultura de segurança do paciente dentro da instituição. Programas de treinamento, capacitação e conscientização da importância da correta utilização do checklist de cirurgia segura é fundamental para sensibilizar a equipe multidisciplinar do centro cirúrgico de que essa prática salva-vidas. 


\section{REFERÊNCIAS}

1. Reis CT, Martins M, Laguarda J. A segurança do paciente como dimensão da qualidade do cuidado de saúde: um olhar sobre a literatura. Ciênc. Saúde Coletiva[periódico na internet] 2013 [acessado 2021 maio 27];18(7).Doi:https://doi.org/10.1590/S1413-81232013000700018

2. Fonseca RMP, Peniche ACG. Enfermagem em centro cirúrgico: trinta anos após criação do sistema de assistência de enfermagem Perioperatória. Acta paul. Enferm. [periódico na internet] 2009[acessado 2021 maio 27];22(4). Disponível em: https://www.scielo.br/j/ape/a/Lyq5Vw48j4gvgcBQMNzTcFn/?lang=pt

3. Elias ACGP, Schmidt DRC, Yonekura CSI, Dias AO, Ursi ES, Silva RPJ et al. Avaliação da adesão ao check-list de cirurgia segura em hospital universitário público.Rev. Sobecc[periódico na internet]2015[acessado 2021 maio 27];20(3): 128-133. Disponível em: http://files.bvs.br/upload/S/1414-4425/2015/v20n3/a5166.pdf

4. Oliveira RM, Leitão IMTA, Silva LMS, Figueiredo SV, Sampaio RL, Gondim MM. Estratégias para promover segurança do paciente: da identificação dos riscos as práticas baseadas em evidências.Esc.Anna Nery Rev. Enferm [periódico na internet] 2014[acessado 2021 maio 27];18(1):122-129.Disponível em:https://pesquisa.bvsalud.org/portal/resource/pt/lil-704662

5. Freitas MR, Antunes AG, Lopes BNA, Fernandes FC, Monte LC, Gama ZAS. Avaliação da adesão ao check-list de cirurgia segura da OMS em cirurgias urológicas e ginecológicas, em dois hospitais de ensino de Natal, Rio Grande do Norte, Brasil.Rev. Sobecc[periódico na internet]2019[acessado 2021 maio 27];24(1):22-30. Disponível em: https://revista.sobecc.org.br/sobecc/article/view/437

6. Pancieri AP, Carvalho R, Braga EM. Aplicação do check-list para cirurgia segura: relato de experiência. Rev. Sobecc[periódico na internet]2014[acessado 2021 maio 27];19(1):26- 
33.Disponível em: https://revista.sobecc.org.br/sobecc/article/view/50

7. Amaya MR, Mazieiro ECS, Grittem L, Cruz EDA. Análise do registro e conteúdo de checklist para cirurgia segura. Esc. Anna Nery RevEnferm[periódico na internet] 2015[acessado 2021 maio 27];19(2):246-251. Disponível em: https://www.scielo.br/pdf/ean/v19n2/14148145-ean-19-02-0246.pdf

8. Brousselle A, Champagne F, Contandriopoulos AP, Hartz z. Avaliação: conceitos e métodos. Ciênc. Saúde Coletiva [periódico na internet]2011[acessado 2021 maio 27];17(4):1091-1092. Disponível em: https://www.redalyc.org/articulo.oa?id=63023349030

9. Pedron JA. Metodologia cientifica: auxiliar no estudo da leitura e da pesquisa. Brasília-DF, Brasil, 2001.

10. Correggio TC, Amante LN, Barbosa SFF. Avaliação da cultura de segurança do paciente em centro cirúrgico. Rev. Sobecc[periódico na internet] 2014 [acessado 2021 maio 27];19(2):6773. Doi: http://doi.editoracubo.com.br/10.4322/sobecc.2014.012

11. Roscani ANCP, Ferraz EM, Filho AGO, Freitas MIP. Validação de check-list cirúrgico para prevenção de infecção de sítio cirúrgico. Acta Paul. Enferm.[periódico na internet] 2015 [acessado 2021 maio 27];28(6):553-565. Doi:https://doi.org/10.1590/1982-0194201500092

12. Ribeiro L, Fernades GC, Souza EG, Souto LC, Santos ASP, Bastos RR. Checklist de cirurgia segura: adesão ao preenchimento, inconsistências e desafios.Rev. Col. Bras. Cir[periódico na internet]2019[acessado 2021 maio 27];46(5): e20192311. Doi:https://doi.org/10.1590/0100$\underline{6991 \mathrm{e}-20192311}$

13. Pancieri AP, Santos BP, Avila MAG, Braga EM. Checklist de cirurgia segura: análise da segurança e comunicação das equipes de um hospital escola.Rev. Gaúcha Enferm.[periódico na internet]2013 [acessado 2021 maio 27];34(1):71-78. Doi: https://doi.org/10.1590/S1983$\underline{14472013000100009}$ 